\title{
BMJ Open Barriers and facilitators in the integration of oral health into primary care: a scoping review
}

\author{
Hermina Harnagea, ${ }^{1}$ Yves Couturier, ${ }^{2}$ Richa Shrivastava, ${ }^{3}$ Felix Girard, ${ }^{3}$ \\ Lise Lamothe, ${ }^{1,4}$ Christophe Pierre Bedos, ${ }^{5}$ Elham Emami ${ }^{1,3,4,5}$
}

To cite: Harnagea $\mathrm{H}$, Couturier Y, Shrivastava $\mathrm{R}$, et al. Barriers and facilitators in the integration of oral health into primary care: a scoping review. BMJ Open 2017;7:e016078. doi:10.1136/ bmjopen-2017-016078

- Prepublication history for this paper is available online To view these files, please visit the journal online (http://dx.doi. org/10.1136/bmjopen-2017016078).

Received 25 January 2017 Revised 1 June 2017 Accepted 2 June 2017

\section{CrossMark}

${ }^{1}$ School of Public Health, Université de Montréal, Montréal, Québec, Canada ${ }^{2}$ School of Social Work, Université de Sherbrooke, Sherbrooke, Québec, Canada ${ }^{3}$ Faculty of Dental Medicine, Université de Montréal, Montréal, Québec, Canada ${ }^{4}$ Public Health Research Institute, Université de Montréal, Montréal, Québec, Canada

${ }^{5}$ Faculty of Dentistry, McGill University, Montréal, Québec, Canada

Correspondence to

Dr Elham Emami;

elham.emami@umontreal.ca

\section{ABSTRACT}

Objective This scoping study has been conducted to map the literature and provide a descriptive synthesis on the barriers and facilitators of the integration of oral health into primary care.

Methods Grounded in the Rainbow conceptual model and using the Levac et al six-stage framework, we performed a systematic search of electronic databases, organisational websites and grey literature from 1978 to April 2016. All publications with a focus on the integration of oral health into primary care were included except commentaries and editorials. Thematic analyses were performed to synthesise the results.

Results From a total of 1619 citations, 58 publications were included in the review. Barrier-related themes included: lack of political leadership and healthcare policies; implementation challenges; discipline-oriented education; lack of continuity of care and services and patients' oral healthcare needs. The facilitators of integration were supportive policies and resources allocation, interdisciplinary education, collaborative practices between dental and other healthcare professionals, presence of local strategic leaders and geographical proximity.

Discussion and public health implications This work has advanced the knowledge on the barriers and facilitators at each integration domain and level, which may be helpful if the healthcare organisations decide to integrate oral health and dental services into primary care. The scoping review findings could be useful for both dental and medical workforce and allied primary healthcare providers. They could also guide the development of healthcare policies that support collaborative practices and patient-centred care in the field of primary care.

\section{BACKGROUND}

Over the last decades, the concept of integration has been implemented as a multidisciplinary care pathway in many health organisations to increase the effectiveness of care for patients with special clinical needs and problems, such as elders and patients with cognitive or physical disabilities. ${ }^{1-6}$ The integrated care approach has mainly emerged in primary healthcare settings to provide and maintain universal access to a

\section{Strengths and limitations of this study}

- This scoping review identified the barriers and facilitators of the integration of oral health through a comprehensive analysis of the literature using a theoretical framework.

- The implications of these findings will allow the development of targeted strategies that could increase the integration of oral health into primary care by eliminating common barriers and enhancing facilitators.

- The nature of the scoping review did not allow the grading of the evidence since a quality evaluation of the included studies has not been conducted. This could be an objective for a further systematic review.

broad range of healthcare services. However, this patient-centred care model faces challenges and resistance in adoption for some domains or disciplines such as oral health and dentistry. ${ }^{7}$ In fact, the integration of oral health into primary care is still at the stage of initiative in many countries. Recently, the American Academy of Family Physicians has supported the integration of oral health into primary care as delineated by the Oral Health Delivery Framework. ${ }^{8}$ This framework refers to multidisciplinary collaborative practices for risk assessment, oral health evaluation, preventive interventions as well as communication and education. It was developed by an interdisciplinary team of health and oral healthcare providers, representatives of professional associations and public health advocates as well as policy-makers and care consumers. However, this concept is still relatively new and needs to be examined in its comprehensive perspective. As defined by Gröne and Garcia-Barbero, integrated care is 'bringing together inputs, delivery, management and organisation of services related to diagnosis, treatment, care, rehabilitation and health promotion'. ${ }^{9}$ Furthermore, the adoption of integrated care models 
in healthcare systems necessitates identifying barriers, sharing knowledge and delivering necessary information to policy-makers.

As presented in the published protocol, ${ }^{10}$ a comprehensive scoping review funded by the Canadian Institutes for Health Research has been conducted by Emami's research team to answer several research questions on the concept of the primary oral healthcare approach. The scoping review findings have been divided and prepared for presentation into two publications. This paper presents specifically the results on the barriers and facilitators. The findings in regard to policies, applied programmes and outcomes will be presented in the subsequent publication.

\section{METHODS}

The method outlined by Levac et $a l^{11}$ an extension of the Arksey and O'Malley scoping review method, ${ }^{12}$ has been used to conduct the review. Since the methods employed in this scoping review have been presented in detail previously, ${ }^{10}$ they are described only briefly here. The Levac et al methodological framework comprises six stages: (1) identifying the research question, (2) searching for relevant studies, (3) selecting studies, (4) charting and collating the data, (5) summarising and reporting the results and 6) consultation with stakeholders to inform the review. ${ }^{11}$

\section{Research question}

The following research question has been formulated for this part of the review: What are the barriers and the facilitators of the integration of oral health into primary care in various healthcare settings across the world?

\section{Search strategy}

A detailed search strategy was designed with the help of an expert librarian at Université de Montreal, using specific MeSH terms and keywords to capture the relevant literature on the topic of interest. We created groupings of keywords and medical subject headings that were combined with the Boolean terms 'OR' and 'AND' and 'NOT'. The search strategy was developed for Medline via Ovid interface (table 1) and was revised for each of the other electronic platforms such as: Ovid (Medline, Embase, Cochrane databases), National Center for Biotechnology Information (PubMed), EBSCOhost (Cumulative Index to Nursing and Allied Health Literature), ProQuest, Databases in Public Health, Databases of the National Institutes of Health (health management and health technology), Health Services and Sciences Research Resources, Health Services Research and Health Care Technology, Health Services Research Information Central, Health Services Research Information Portal, Health Services Technology Assessment Texts and Healthy People 2020. For this last platform, we used the Healthy People Structured Evidence Queries, which are preformulated PubMed searches for Healthy People 2020 (HP2020) objectives. These ongoing updated queries have been developed by experts, librarians and stakeholders in the field of public health to achieve HP2020 objectives to easily search the evidence-based public health literature.

\section{Identifying relevant studies and eligibility criteria}

Publications in English or French from 1978 to April 2016 were reviewed. We included all research studies irrespective of study design in which the integration of oral health into primary care is the primary focus of the publication. We excluded publications such as commentaries, editorials and individual points of view, but we searched their references for the original studies. Two researchers (HH, EE) independently screened the titles and abstracts of each citation and identified eligible articles for full review. Disagreement between reviewers was discussed and resolved by consensus. All potentially relevant studies were retained for full-text assessment. Data extraction was conducted independently by the same reviewers using a data extraction form, designed according to the study's conceptual framework.

\section{Conceptual framework}

The Rainbow model was used as a conceptual model to guide the scoping study. ${ }^{13}$ This model is based on the integrative functions in primary care and includes level-specific domains: clinical integration (micro level), organisational and professional integration (meso level) and system integration (macro level). Furthermore, in this multilevel model, functional and normative integration assure the link between the other three domains.

\section{Data charting and collating}

To ensure the consistency of the data extraction, this stage was conducted by three reviewers (HH, EE, RS) followed by consensus. The data were classified into two tables, according to the type of the publications: (1) research reports; (2) policies, strategic plans and other relevant publications. In the first step, extracted data and related meaning units were grouped into two categories: barriers and facilitators. According to Tesch (1990), a meaning unit is 'a segment of text that is comprehensible by itself and contains one idea, episode or piece of information'. ${ }^{14}$ Then a constant comparison of the codes was conducted and the themes were identified. In the second step, these categories were divided into specific levels and domains according to the study's conceptual framework. At this stage, a triangulation was conducted by the scoping review team (HH, EE, RS, FG, YC, LL, CB) and themes were discussed and revised.

\section{Summarising and reporting the results}

A qualitative approach was used to synthesise the study's findings. This involved a descriptive and thematic analysis of the results based on the conceptual framework.

\section{Stakeholder consultations}

We engaged the knowledge users and stakeholders in the entire process of the review through preliminary reviews 
Table 1 Medline search strategy

\begin{tabular}{ll}
\hline$\#$ & Searches \\
\hline 1 & exp Dental Health Services/ \\
\hline 2 & Oral Health/ \\
\hline 3 & Dentistry/ \\
\hline 4 & Oral Medicine/ \\
\hline 5 & exp Preventive Dentistry/ \\
\hline 6 & exp Dental Facilities/ \\
\hline 7 & exp Diagnosis, Oral/ \\
\hline 8 & Stomatognathic Diseases/ \\
\hline 9 & exp Mouth Diseases/ \\
\hline 10 & exp Tooth Diseases/ \\
\hline 11 & Pediatric Dentistry/ \\
\hline 12 & exp Dentists/ \\
\hline 13 & Community Dentistry/ \\
\hline 14 & (dentist or stomatogy or DentalProphylaxis or Fluoridation or Oral Hygiene oral Heath or Dental Facities or Dental
\end{tabular}

14 (dentist* or stomatology or Dental Prophylaxis or Fluoridation or Oral Hygiene or Oral Health or Dental Facilities or Dental

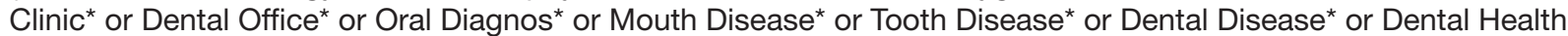
Service* or Dental Service* or pedodontics).mp. [mp=title, abstract, original title, name of substance word, subject heading word, keyword heading word, protocol supplementary concept word, rare disease supplementary concept word, unique identifier]

151 or 2 or 3 or 4 or 5 or 6 or 7 or 8 or 9 or 10 or 11 or 12 or 13 or 14

16 exp Primary Health Care/

17 Primary Care Nursing/

18 Primary Nursing/

19 Physicians, Primary Care/

20 (Primary care or Primary health care or Primary healthcare or Primary Nursing).mp. [mp=title, abstract, original title, name of substance word, subject heading word, keyword heading word, protocol supplementary concept word, rare disease supplementary concept word, unique identifier]

2116 or 17 or 18 or 19 or 20

22 exp 'Delivery of Health Care, Integrated'/

23 exp Community Health Services/

24 (community care or community health care or community healthcare).mp. [mp=title, abstract, original title, name of substance word, subject heading word, keyword heading word, protocol supplementary concept word, rare disease supplementary concept word, unique identifier]

2522 or 23 or 24

26 Community Integration/

27 systems integration/

28 (Integrat* or Interprofessional or multidisciplin* or interdisciplin* or cooperat* or collaborat* or coordination*).mp. [mp=title, abstract, original title, name of substance word, subject heading word, keyword heading word, protocol supplementary concept word, rare disease supplementary concept word, unique identifier]

29 ((Cross or multi or inter) adj (profession* or Disciplin*)).mp. [mp=title, abstract, original title, name of substance word, subject heading word, keyword heading word, protocol supplementary concept word, rare disease supplementary concept word, unique identifier]

3026 or 27 or 28 or 29

$31 \quad 15$ and 21 and 30

32 limit 31 to (English or French)

33 (15 and 25 and 30) not 31

34 limit 33 to (English or French) 


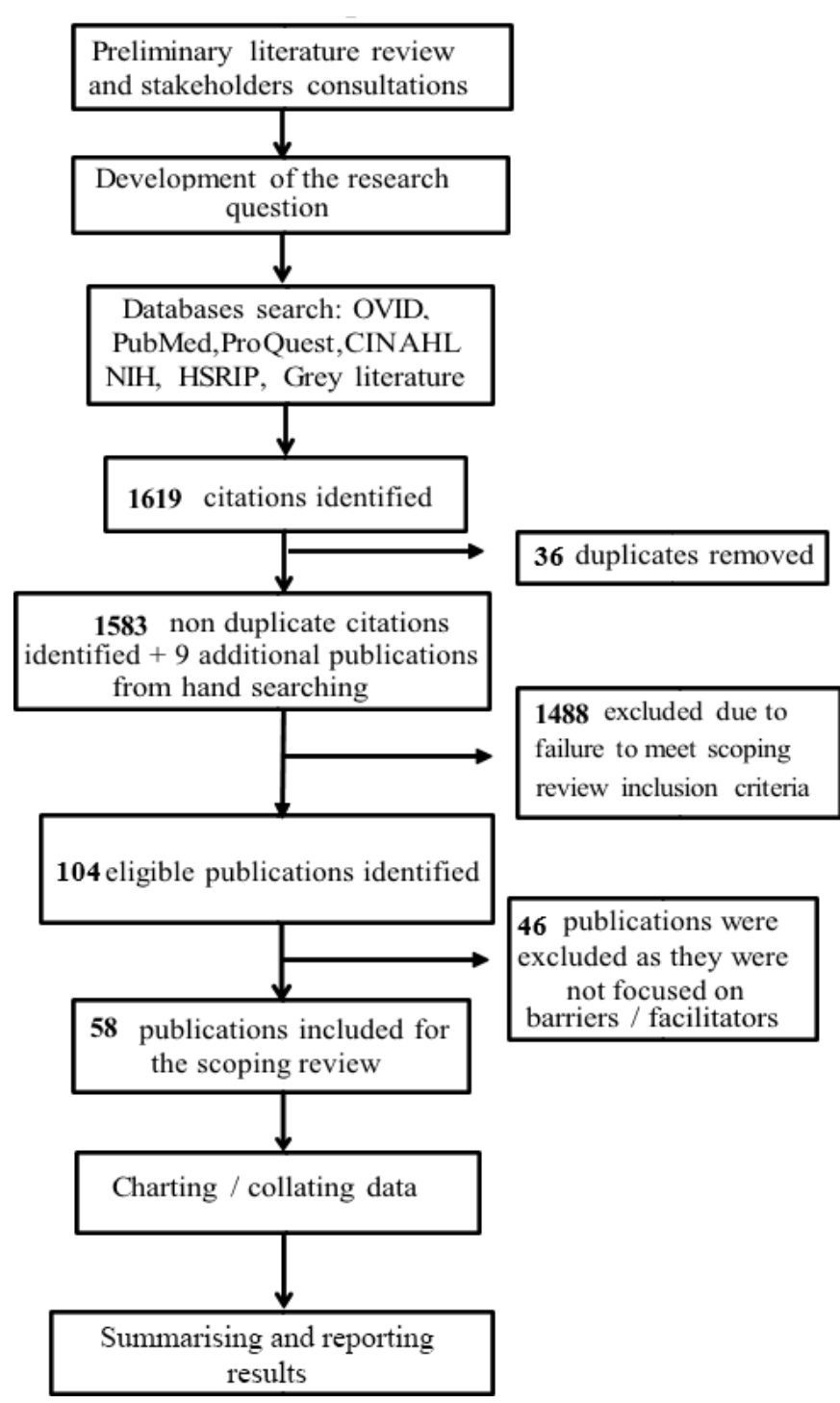

Figure 1 Flow chart of the scoping review.

of a few published articles, as well as discussions on the study research question. The stakeholders included representatives of academic healthcare organisations, policy decision-makers and primary healthcare professionals working in rural and remote communities, as well as patients' representatives.

\section{RESULTS}

\section{Characteristics of the publications}

The databases and grey literature searches yielded 1619 records (figure 1). After removal of duplicates, 1583 publications went through title and abstract screening, of which 95 were included for full review. After adding nine publications from the hand search of references, a total of 104 articles were included in the final analysis. Among the total reviewed articles, 58 publications (tables 2 and 3) reported on the barriers and/or facilitators of oral health integration into primary care. These publications were from 18 countries across the world: the USA, Australia, Canada, France, Sweden, Norway, Switzerland, Nepal, Bangladesh, Indonesia, Tanzania, Nigeria, Thailand, Peru, Brazil, New Zealand, the UK and Iran.

The majority of research studies were published in the last decade and were conducted in the USA. Table 2 presents the characteristics of the selected original research studies $(\mathrm{n}=37){ }^{15-51}$ The research studies included pilot and demonstration projects, qualitative and quantitative studies. The latter included two randomised controlled trials (RCTs). The publications in regard to policy analyses/white papers, oral healthcare programme descriptions $(\mathrm{n}=21)$ are presented in table $3 .^{52-72}$

The publications reported barriers and facilitators on the three levels of integration as described by Leutz et $a l^{3}$ : linkage $(\mathrm{n}=41)$; coordination $(\mathrm{n}=11)$ and full integration $(n=6)$. Only seven publications from three countries reported on the long-term barriers of fully integrated models of primary oral care. ${ }^{15} 172746657072$ Furthermore, the types of integration reported in the literature were mostly at the linkage level and included screening to identify emerging needs, understanding and responding to the special needs of identified vulnerable population groups such as children and elders, referrals and follow-up and providing information to patients.

\section{Themes}

A total of 10 themes and 9 subthemes at the macro, meso and micro level emerged from the review. These themes covered all the domains found in the theoretical model. The most frequently reported barrier was related to primary healthcare providers' competencies at the micro level and in the domain of clinical integration. The two other most reported barriers were the low political priority in the system integration domain, at the macro level, as well as the lack of funds in the organisational integration domain, at the meso level. The most frequently reported facilitators included collaborative practices in the functional domain and financial support in the system integration domain, at the macro level.

\section{Barriers in the integration of oral health into primary care Lack of political leadership and healthcare policies}

Lack of political leadership, poor understanding of the oral health status of the population and low prioritisation of oral health on the political agenda as well the absence of appropriate oral health policies were identified as barriers for integrated care at the macro level. ${ }^{19} 212225324048-5172$ Insurance policies and separate medical and dental insurance realms were found detrimental to the coordination of services among medical and dental providers in the functional domain. ${ }^{40} 5359$ Furthermore, in many countries, the professional legislation policies did not allow the delivery of preventive oral healthcare by non-dental professionals, and this operates as a barrier for integrated care. $^{18192540}$ 


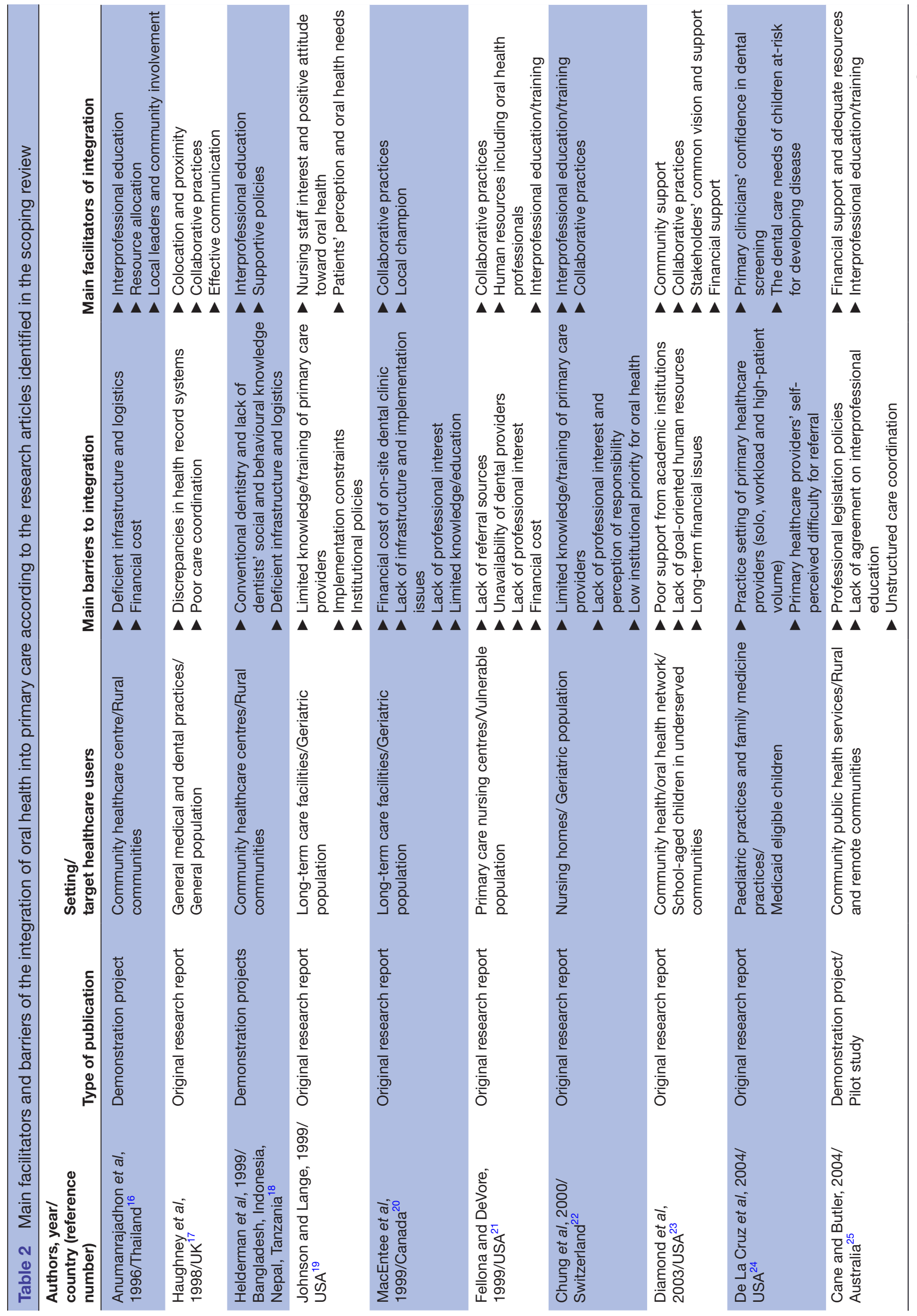




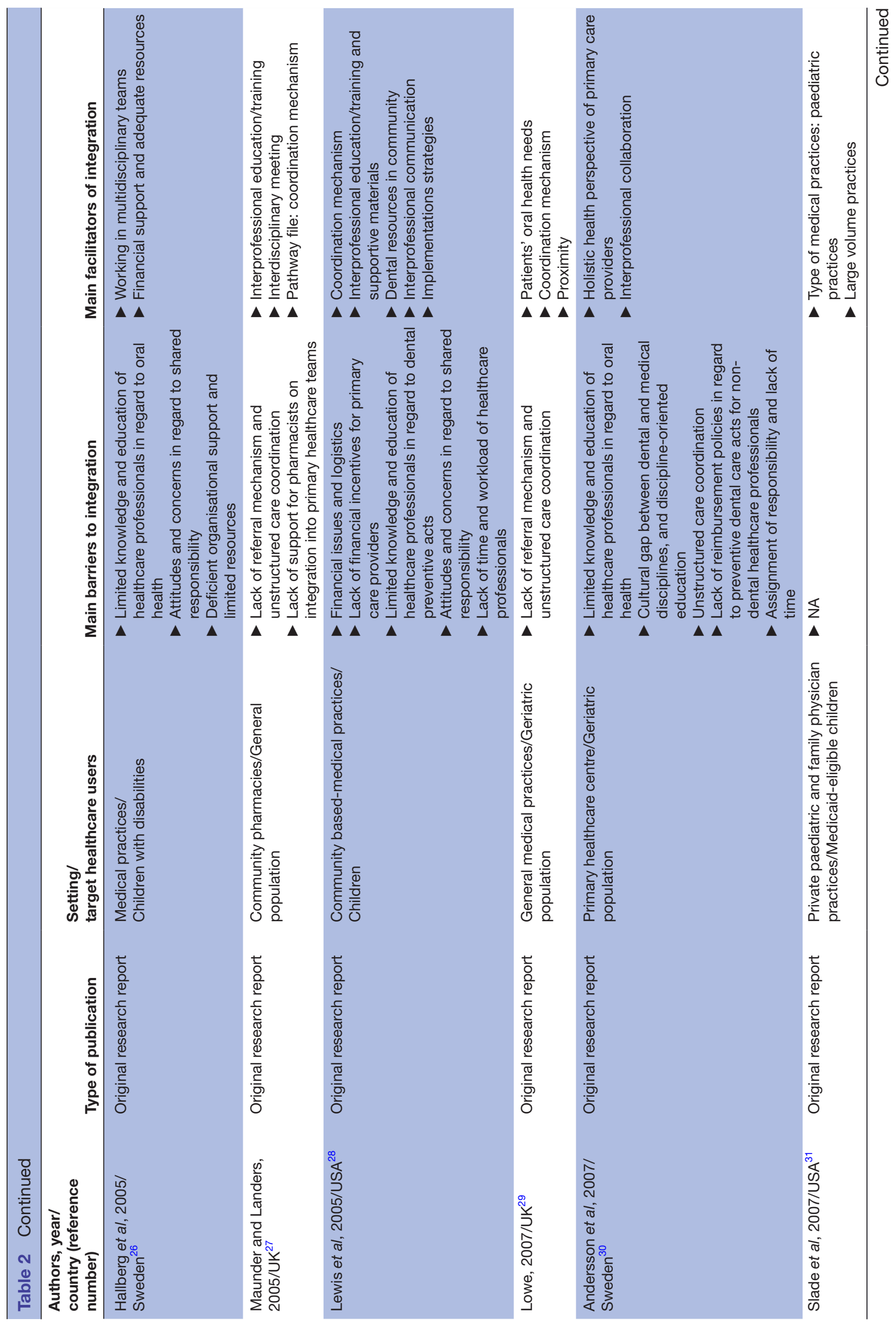

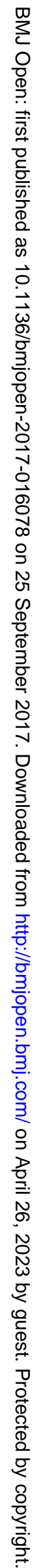




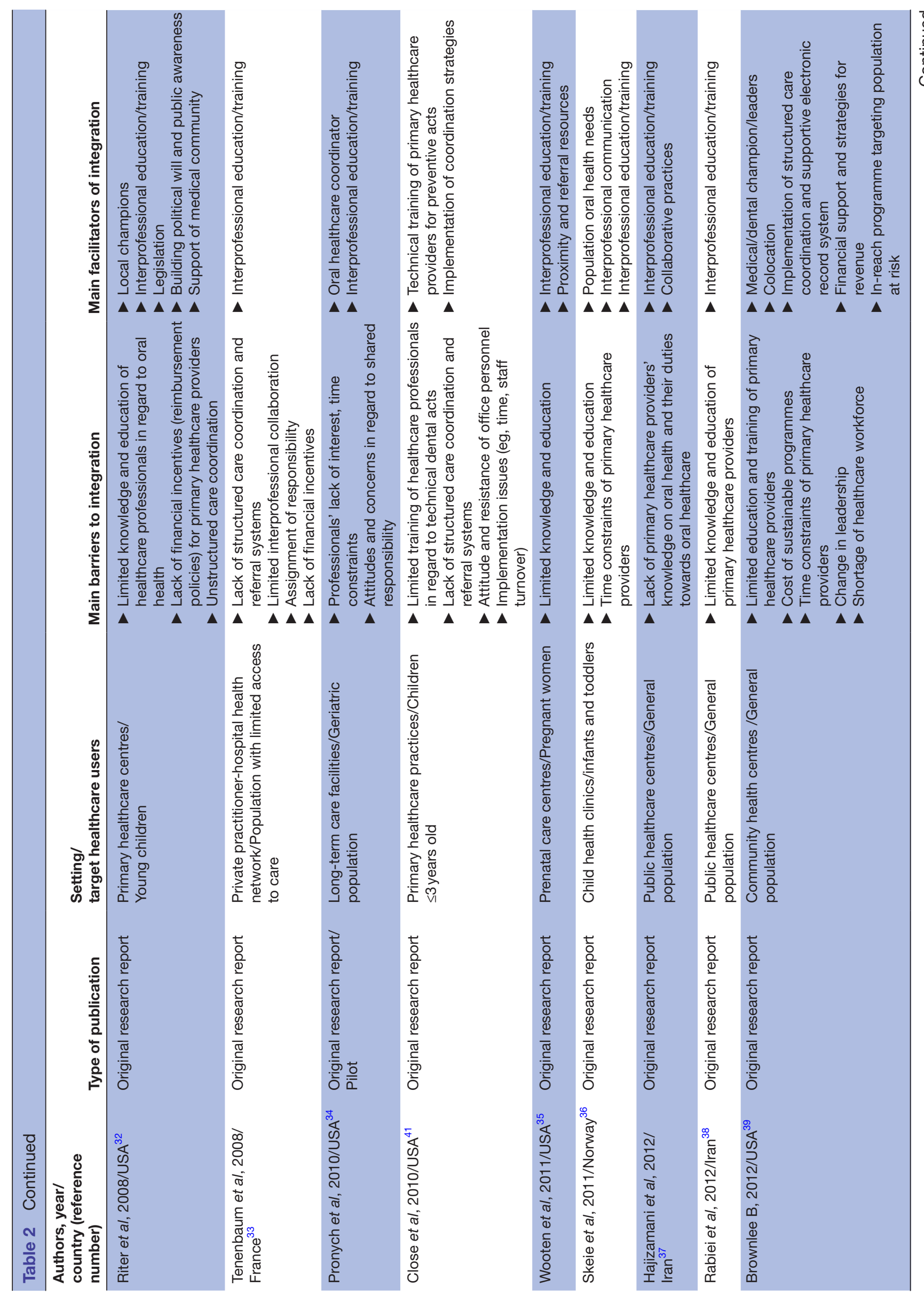




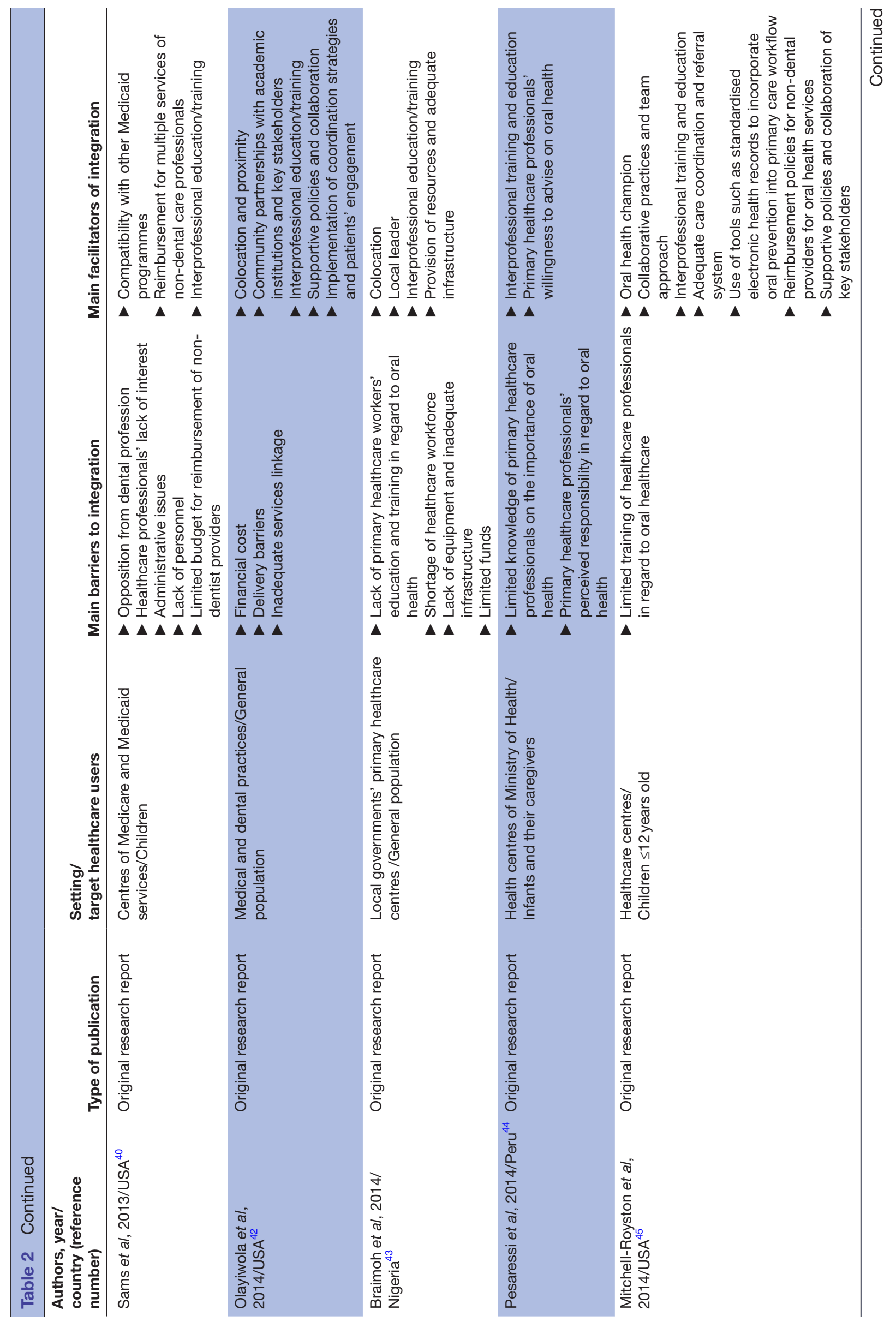




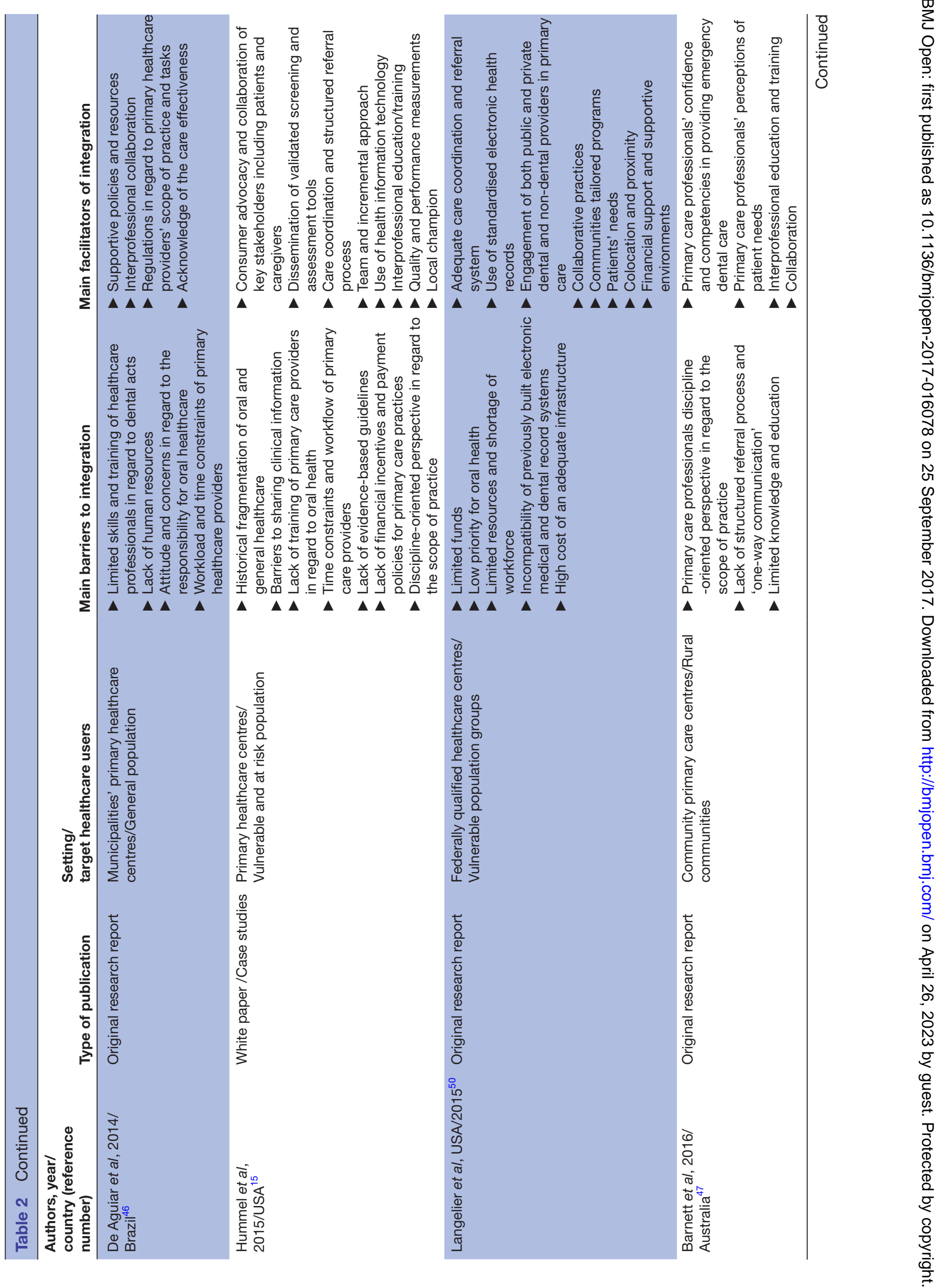




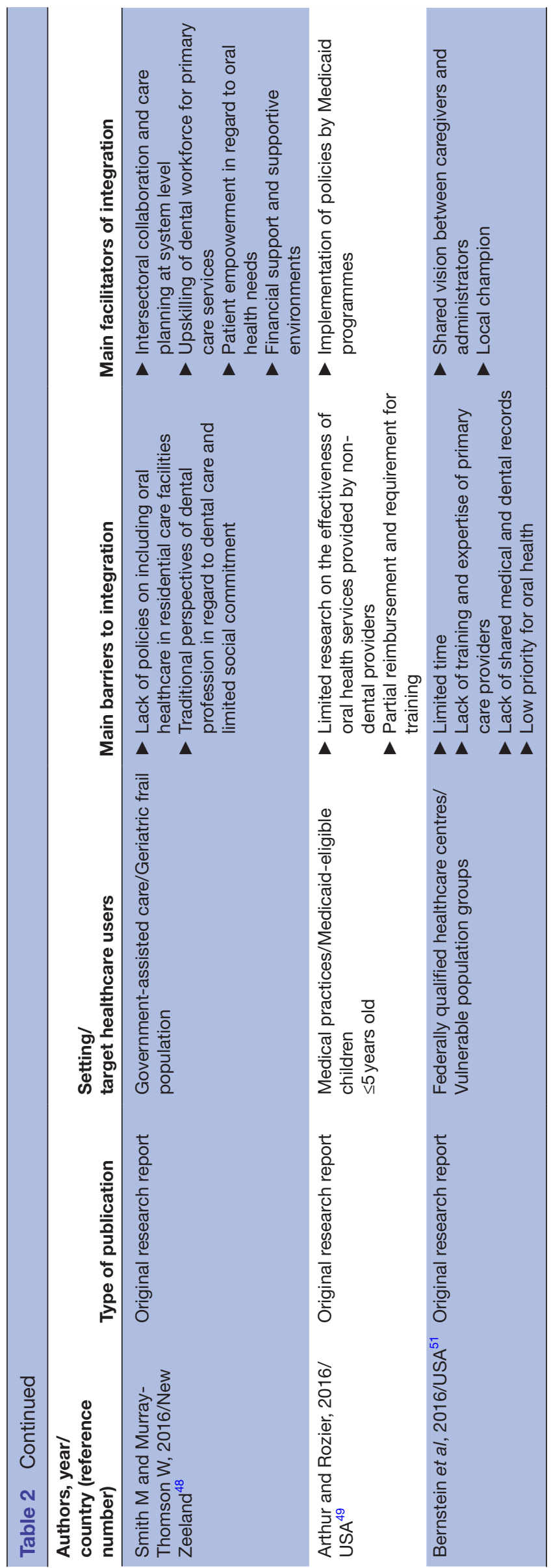

Implementation challenges

The cost of integrated services, human resources issues and deficient administrative infrastructure were reported as major barriers in implementation of oral health integrated care at the meso and macro levels. ${ }^{162021262833424348}$ The challenges to ensure the economic stability of programmes targeting oral health in primary care and the high cost of equipment maintenance were frequently reported as barriers. ${ }^{66}{ }^{69}$ Many studies were in accordance with the fact that workload of personnel, staff turnover, time constraints and scarcity of various trained human resources such as care coordinators, public health workforce and allied dentists were important barriers to oral health integrated care. ${ }^{15242830343639414651545766}$ Moreover, recruitment and retention of dental and non-dental staff were considered challenging, mostly due to the limited number of professionals interested in working in primary integrated clinics and shortage of dentists in rural and remote regions. ${ }^{48371}$

Deficient administrative infrastructure such as the absence of dental health records in medical records, cross-domain interoperability and domain-specific act codes were considered as a contributor to the general perception of dental care as an 'optional' service, hindering medical professionals from performing basic dental services. ${ }^{59} 6769$

\section{Discipline-oriented education and lack of competencies}

At the meso level, lack of interprofessional education and focusing on discipline-oriented training in health were identified as obstacles to integrated care in many studies. ${ }^{18-20} 22$ 26-28 3032 35-39 41 43-48 50 515466 This barrier was translated at the micro level as lack of competencies. Knowledge, attitudes and skills were the most reported meaning units of competencies of primary healthcare providers, as defined by Bloom and Krathwohl. ${ }^{74}$ The lack of knowledge in regard to integrated care practices was identified for both dental and non-dental care providers. For instance, a study conducted in the USA showed that paediatricians with a low level of competencies had adopted oral healthcare into their routine practice five times less than those with a higher level. ${ }^{24}$ Besides, qualitative studies conducted in Sweden, France and Brazil found various attitudes towards integrated care in both dental and medical healthcare teams, in terms of professional interests, shared tasks and responsibility. ${ }^{26} 3346$ Chung et al found that $33 \%$ of the physicians in a longterm care facility declared carrying out a systematic examination of the oral cavity, while the others expressed feelings of illegitimacy and considered oral health as an exclusive dentist domain. ${ }^{22}$ Moreover, and contrary to nursing personnel in a long-term care facility, only a minority of the physicians stressed that oral healthcare of the residents should be carried out on site by a dentist. ${ }^{20}$

Lack of continuity of care and services

The theme continuity of services included three subthemes: unstructured mechanism for care 


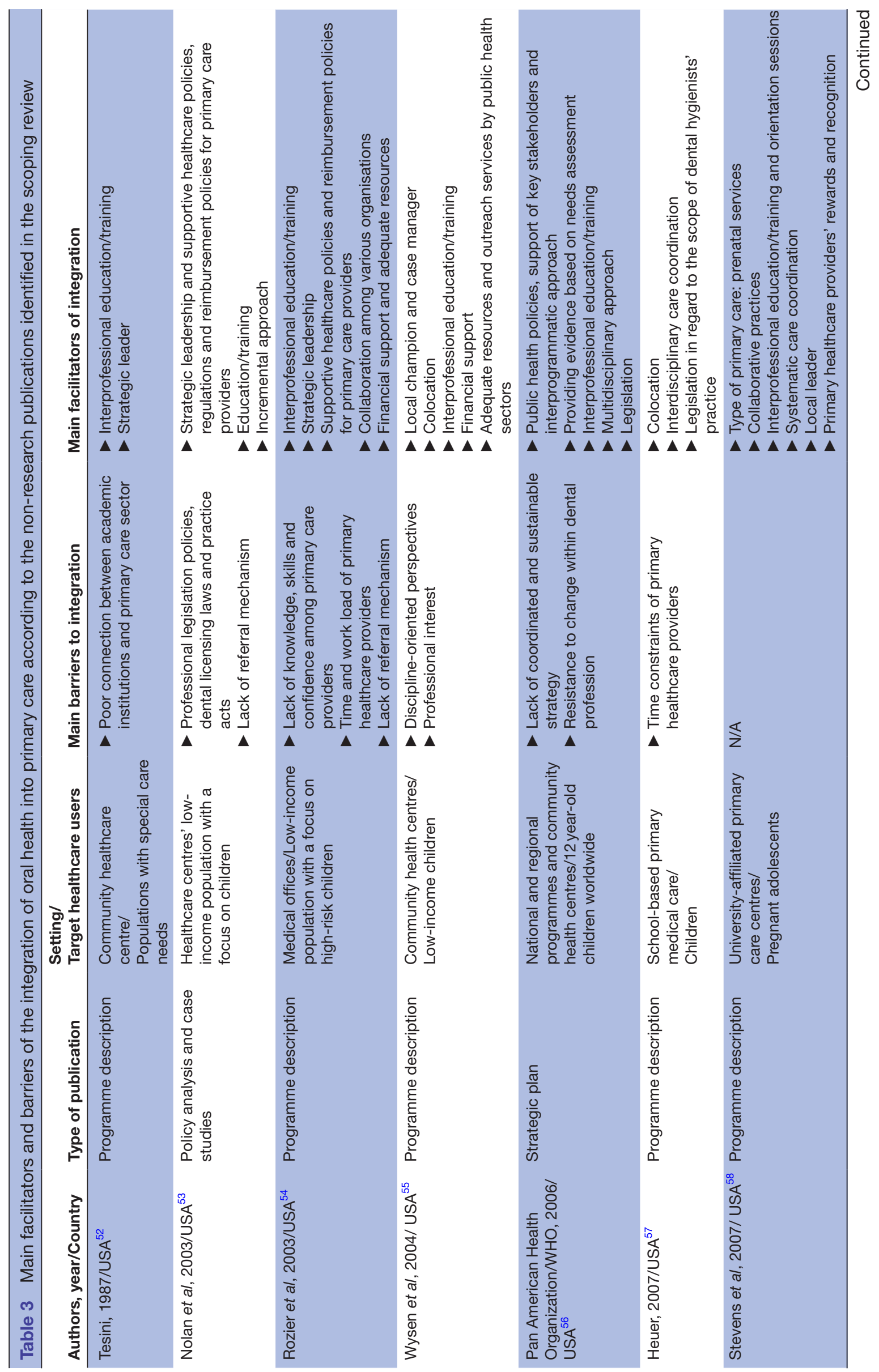




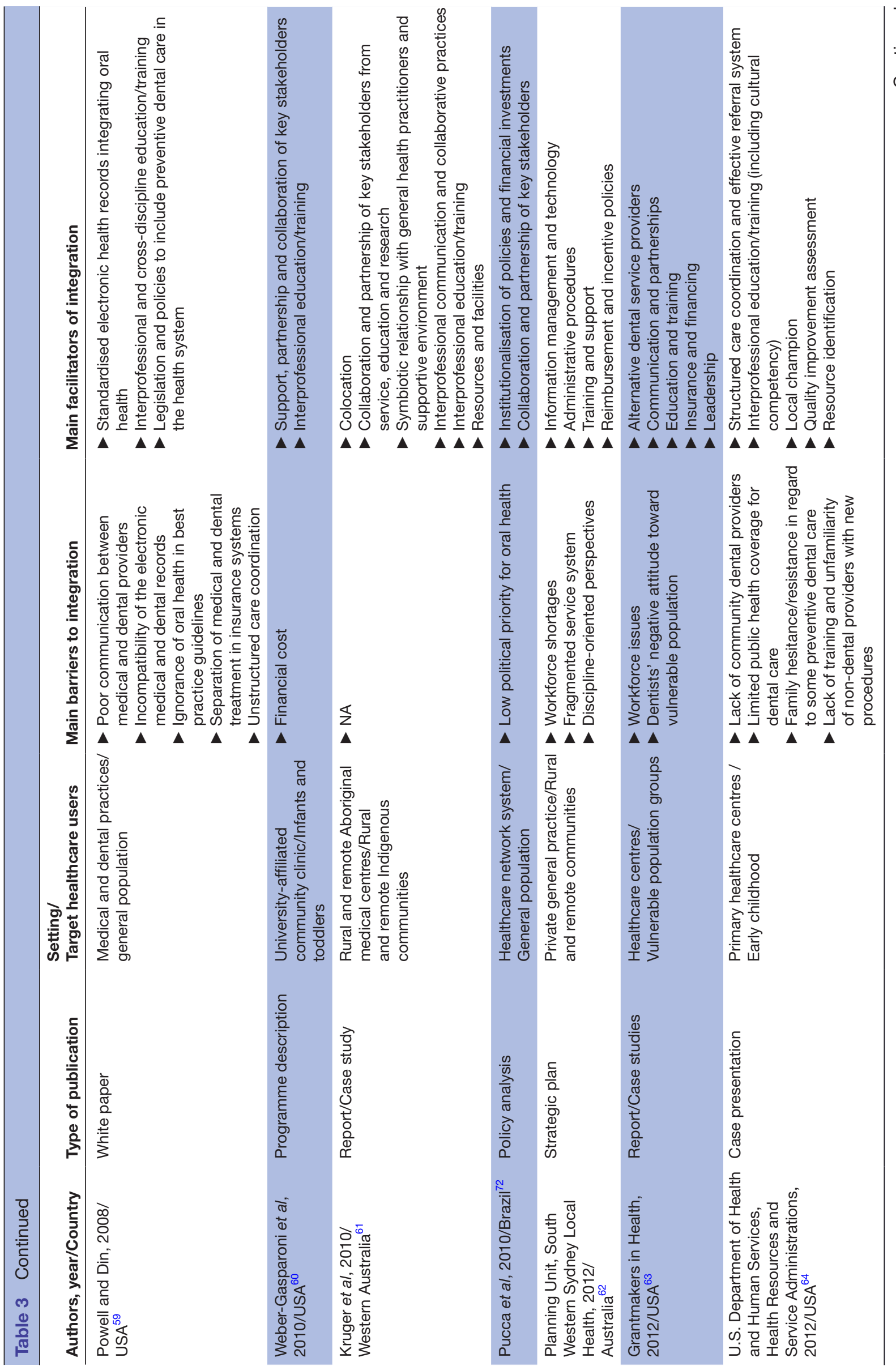

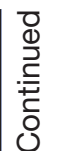




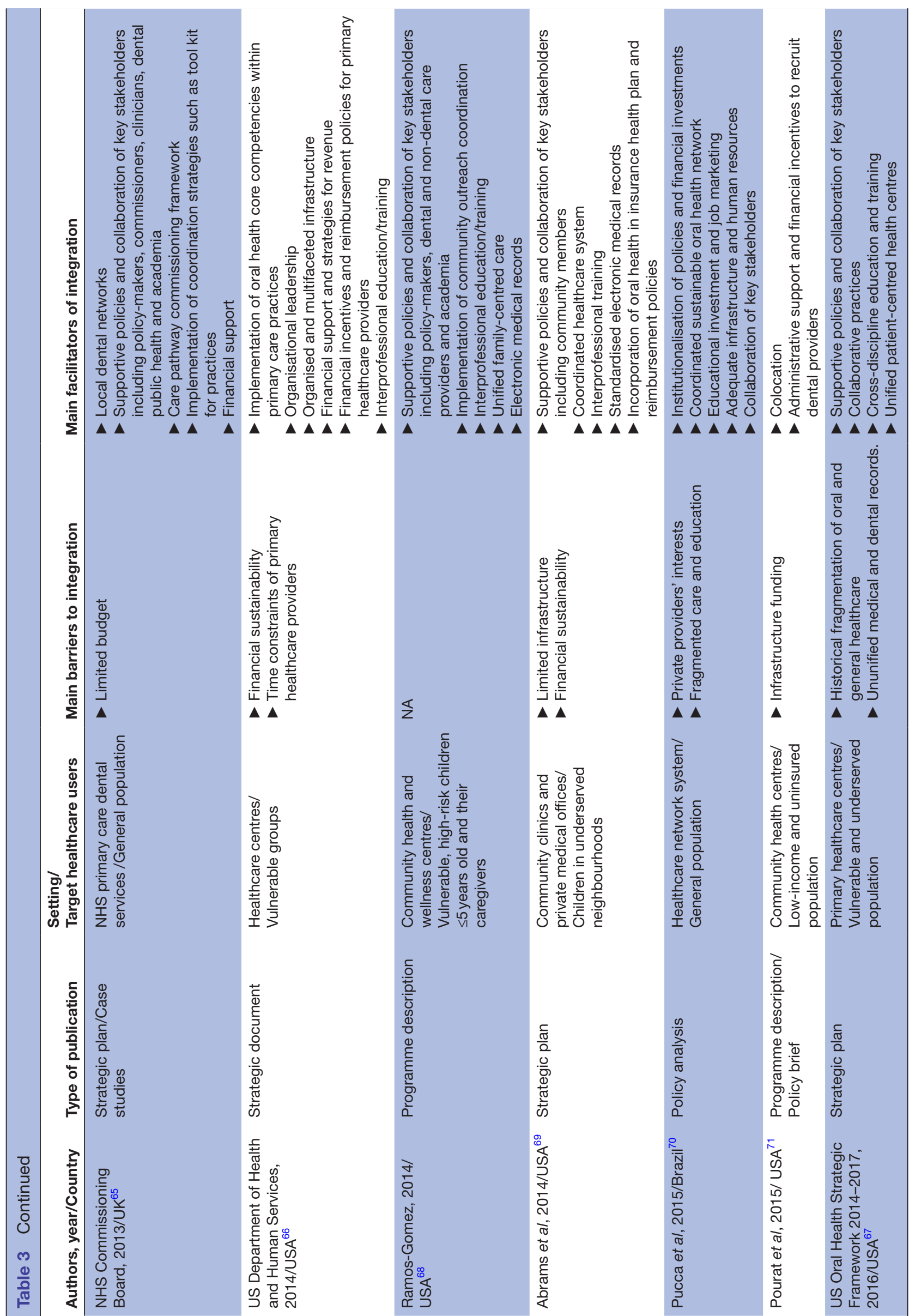

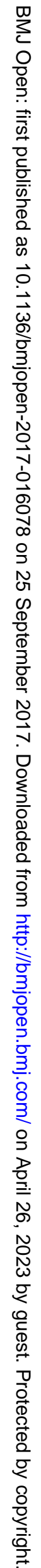


coordination at the micro level and lack of practice guidelines and types of practice at the meso level. Discontinuity in the integrated care process was associated with poor referral systems, deficient interface and poor connection between public health section, primary care and academic institutions. ${ }^{21} 2729323341475354$ Furthermore, practice types such as in silo practices and contract-based services were reported as barriers for linkage, coordination and integration of services. ${ }^{152}$ Some studies showed that solo practices and practices with specific clienteles such as infants and toddlers had lower referral rates to dentists than polyclinics with various clienteles. ${ }^{24} 54$

\section{Patient's oral healthcare needs}

The review of publications revealed that patients' decision to accept or refuse integrative care was mainly based on their need perception rather than the assessment of healthcare providers. ${ }^{19} 242936$ In an RCT conducted by Lowe $e t a l$, current dental problem and not having a regular dentist were the significant predictors for consultation with a non-dental primary care provider. ${ }^{29}$ Patients' problems seem to motivate confident practitioners to provide oral healthcare. ${ }^{2647}$

\section{Facilitators of the integration of oral health into primary care Supportive policies and resources allocation}

Publications on policies and successful integrated programmes highlighted the importance of financial support from governments, stakeholders and non-profit organisations at the macro level. ${ }^{15} 161832394245465354$ Furthermore, several governmental strategic plans highlighted that partnerships and common vision among governments, communities, academia, various stakeholders and non-profit organisations can act as a facilitator to integration of oral health into primary care in the normative domain. ${ }^{56656769}$ Healthcare policies such as Arizona Hygiene Affiliated Practice Act and Medicaid, reimbursements to trained primary care providers for oral screening, patient education and fluoride varnish applications acted as facilitators to the integration of oral health into primary care in the USA. ${ }^{40} 57$ In Brazil, prioritisation of deployment of the National Oral Health Policy by the federal government demonstrated greater integration of oral healthcare in the unified health system, with coverage for access to oral health for the Brazilian population having grown significantly since 2004. . $^{72}$

\section{Interprofessional education}

Several studies revealed that non-dental professionals agreed on interprofessional education, showing higher willingness to include oral health education in their job schedule and to undertake further training on oral health. ${ }^{25} 2728$ 30-32 35-38 40 42-44 $46475254-5658$ 60-63 66-68 Training of paediatricians, family and primary care physicians and community health providers in a preventive dentistry programme in North Carolina (Into the Mouths of Babes), in Seattle (Kids Get Care) and in Washington led to the integration of preventive dental services into their practices. ${ }^{285455}$

\section{Collaborative practices}

This theme included three subthemes: perceived responsibility and role identification, case management and incremental approach. Although many studies reported a lack of oral health knowledge among various healthcare providers, it was also reported that understanding their role in providing oral healthcare could act as a facilitator to engage them in integrated oral healthcare services. $^{19-23} 262730424446-4851586065-69$ According to some studies conducted in North Carolina and Peru, primary care physicians and nurses were able to identify their role and assumed their responsibility in taking care of the oral health of their patients. ${ }^{44}$ Besides, integrated primary care in Glasgow reported positive response on the part of professionals towards joint-work practices. ${ }^{17}$

Two pilot studies reported that appropriate case management, including choice and flexibility in service delivery at multiple levels (administrative and/or clinical) could lead to effective coordination and consistency between oral health and other healthcare services. ${ }^{16} 25$ Some programmes such as the Neighborhood Outreach Action for Health (NOAH) oral health programme in Arizona showed success in primary care teamwork when sharing oral healthcare responsibilities with nurses, medical assistants and other members of the team. ${ }^{57}$ This success relies on an effective coordinated care and strengthening of referral systems, communication among healthcare workers, as well as task-shifting strategies. ${ }^{157-29} 3941424550575864-66$ The incremental approach was suggested as a successful strategy for integration of oral health into primary care. ${ }^{153}$ This approach allowed gradual modification in the workflow based on staff experience and preference.

\section{Local strategic leaders}

Results of studies conducted in the USA and some developing countries highlighted the strategic role of the local leader in building teamwork and communities' capacities in the integration of oral health into primary care. $^{15} 16193238394551556364$ In the Rochester Adolescent Maternity Programme, for instance, registered nurses were found as 'drivers' in promoting oral health by assessing patients' dental needs and managing their consultations and referral. ${ }^{58}$ Similarly, an oral health coordinator in a pilot project in New Hampshire was identified as a linkage facilitator between nursing and dental human resources. ${ }^{34}$

\section{Proximity}

Geographical proximity or colocation of dental and medical practices were reported as the main facilitators for interdisciplinary collaboration in various communities. ${ }^{17424350}$ Healthcare professionals have shown interest in the colocation model since it is the first step to merge primary care and dental care and allows establishing a 
relationship among the healthcare workforce, showing promising results in the delivery of efficient care addressing both the medical and oral health needs of patients. 55576171

According to Wooten et $a l^{35}$ nurses and certified midwives were more likely to adopt preventive measures and refer patients for specialised care if they had a dental clinic in the primary practice setting.

\section{DISCUSSION}

Fragmentation in primary healthcare may put at risk vulnerable patients with chronic or acute health problems such as oral health diseases. ${ }^{175} 76$ However, the integration of oral health into primary care is still at an emerging stage in many countries around the world. Healthcare policy-makers and organisations need high-quality evidence and information to assess their own process gaps and make decisions on its implementation. ${ }^{77}$ Despite the large number of publications on primary healthcare integration, a number of knowledge gaps exist in the domain of oral healthcare integration. To our knowledge, this is the first scoping review aimed at synthesising influential factors in the integration of oral health into primary care using a theoretical model of integration. In fact, the concept of integration is complex and needs to be analysed in a multilevel perspective. In this study, we used the Rainbow model of integrated care to conduct the thematic analysis. ${ }^{13}$ This framework provided a valuable lens to identify level-specific and domain-specific barriers and facilitators across publications. It allows for a better understanding of the inter-relationships among the dimensions of integrated care from a primary care perspective.

The results of the present scoping review are in line with publications on the challenges faced in the implementation of integrated care. ${ }^{78-81}$ Common barriers such as the absence of healthcare policies and supporting strategies, inadequate interdisciplinary training and workload increase seem to depend on both contextual and individual factors rather than the discipline itself. ${ }^{78-81}$ However, in this study we identified a discipline-specific barrier: perception of oral healthcare needs. Some publications reported that patients and most of the primary healthcare providers did not attribute value to continuity of care in the field of oral health because oral health conditions are rarely life threatening. ${ }^{2633} 47$ This aspect, which could be critical from the lens of dental professionals, may be explained by lack of knowledge and awareness of the impact of oral health on general health and well-being and could help explain the fact that oral health is seldom on the political agenda. Interprofessional education and collaboration could be effective in raising awareness on the importance of oral health and its integration into primary care. However, recent studies show that implementation of interprofessional health science curricula is also encountering barriers and requires longterm financial and political supports. ${ }^{82}$ E-health technologies such as online education, electronic health records and web-patient portals could be used to facilitate the implementation of integrated care. ${ }^{83}$

Although some common facilitators such as supportive policies and resource allocation are crucial to mitigate the challenges of integrated care, it seems that the presence of a local leader and proximity have significant impact on making sense of the complex concept of integration, putting collaborative practices in place and involving the stakeholders to make effective and positive change in their organisation.

This scoping review has some strengths and limitations when compared with systematic reviews. Although the scoping review methodology allows the analysis of a broad range of publications, it does not necessitate the quality assessment of publications and grading of evidence. However, scoping reviews provide an avenue for future research and have clinical and public health impact.

\section{CONCLUSION}

The scoping review findings allow better understanding of conceptually grounded barriers and facilitators at each integration domain and level. The most reported barrier themes included primary healthcare providers' competencies at the micro level and in the domain of clinical integration. The most frequently reported facilitators included collaborative practices in the functional domain and financial support in the system integration domain at the macro level. The themes identified here permit the conduct of potential future research and policies to better guide integration of oral healthcare practices between dental and medical workforce and allied primary healthcare providers.

Acknowledgements The authors would like to gratefully acknowledge the help of Mr Dupont Patrice (librarian, Université de Montréal) for the design of the search strategy. We would also like to acknowledge Dr Martin Chartier, Dr John Wootton, Mr Aryan Bayani, Dr Anne Charbonneau, Dr Shahrokh Esfandiari and Dr René Voyer for their collaboration in the study as federal, community and academic organizations representatives. We are grateful for the grant received from the Canadian Institute of Health Research (ClHR) and additional financial support from the FRQ-S Network for Oral and Bone Health Research, Université de Montréal Public Health Research Institute and the Quebec Network of Population Health.

Contributors All authors have made significant contributions to this scoping review. As a principal investigator, EE contributed to the scoping review protocol and secured funds for the study. As a first author, HH collaborated in the protocol development and was involved in all review phases, as well as in the preparation of manuscript draft. RS collaborated in the data extraction and coding. The scoping review team ( $\mathrm{HH}, \mathrm{YC}, \mathrm{RS}, \mathrm{FG}, \mathrm{LL}, \mathrm{EE})$ collectively contributed to the data interpretation, critical revision of the manuscript and its final approval for the publication.

Funding This study is funded by a Knowldege Synthesis Grant from the Canadian Institutes for Health Research (Grant number: KRS-138220).

Competing interests None declared.

Provenance and peer review Not commissioned; externally peer reviewed. Data sharing statement None.

Open Access This is an Open Access article distributed in accordance with the Creative Commons Attribution Non Commercial (CC BY-NC 4.0) license, which permits others to distribute, remix, adapt, build upon this work non-commercially, and license their derivative works on different terms, provided the original work is properly cited and the use is non-commercial. See: http://creativecommons.org/ licenses/by-nc/4.0/ 
(c) Article author(s) (or their employer(s) unless otherwise stated in the text of the article) 2017. All rights reserved. No commercial use is permitted unless otherwise expressly granted.

\section{REFERENCES}

1. Starfield B, Shi L, Macinko J. Contribution of primary care to health systems and health. Milbank Q 2005;83:457-502.

2. Hutchison B, Abelson J, Lavis J. Primary care in Canada: so much innovation, so little change. Health Aff 2001;20:116-31.

3. Béland F, Hollander MJ. Integrated models of care delivery for the frail elderly: international perspectives. Gac Sanit 2011;25 Suppl 2:138-46.

4. Deber RB. Health care reform: lessons from Canada. Am J Public Health 2003;93:20-4.

5. Kodner DL, Spreeuwenberg C. Integrated care: meaning, logic, applications, and implications--a discussion paper. Int J Integr Care 2002;2:e12.

6. Kodner DL, Kyriacou CK. Fully integrated care for frail elderly: two American models. Int J Integr Care 2000;1:e08.

7. Emami E. The integration of oral health in primary care: interdisciplinary research initiative. Mosaic, Canadian Association of Public Health Dentistry. Spring 2015 (7). 2015 http://www.caphd.ca/ sites/default/files/Mosaic Spring 2015.pdf.

8. Phillips K, Hummel J. Oral Health in primary care: a framework for Action. JDR clinical and translational research 2016;1.

9. Gröne O, Garcia-Barbero M; WHO European Office for Integrated Health Care Services. Integrated care: a position paper of the WHO European Office for Integrated Health Care Services. Int J Integr Care 2001;1:e21.

10. Emami E, Harnagea H, Girard F, et al. Integration of oral health into primary care: a scoping review protocol. BMJ Open 2016;6:e013807.

11. Levac D, Colquhoun H, O'Brien KK. Scoping studies: advancing the methodology. Implement Sci 2010:5:69.

12. Arksey H, O'Malley L. Scoping studies: towards a methodological framework. Int J Soc Res Methodol 2005;8:19-32.

13. Valentijn PP, Schepman SM, Opheij W, et al. Understanding integrated care: a comprehensive conceptual framework based on the integrative functions of primary care. Int $J$ Integr Care 2013;13:e010.

14. Tesch R. Qualitative research: analysis, Types and Software tools. Basingstoke, UK: Falmer, 1990.

15. Hummel J, Phillips KE, Holt B, et al. Oral health-an essentia component of primary care-white paper. Safety net medical home initiative. Seattle, WA: Qualis Health, 2015. http://www. safetynetmedicalhome.org/sites/default/files/White-Paper-OralHealth-Primary-Care.pdf. (accessed 23 Oct 2016).

16. Anumanrajadhon T, Rajchagool S, Nitisiri P, et al. The community care model of the Intercountry Centre for Oral Health at Chiangmai, Thailand. Int Dent J 1996;46:325-33.

17. Haughney MG, Devennie JC, Macpherson LM, et al. Integration of primary care dental and medical services: a three-year study. Br Dent $J$ 1998;184:343-7.

18. van Palenstein Helderman W, Mikx F, Begum A, et al. Integrating oral health into primary health care-experiences in Bangladesh, Indonesia, Nepal and Tanzania. Int Dent J 1999;49:240-8.

19. Johnson TE, Lange BM. Preferences for an influences on oral health prevention: perceptions of directors of nursing. Spec Care Dentist 1999:19:173-80.

20. MacEntee MI, Thorne S, Kazanjian A. Conflicting priorities: oral health in long-term care. Spec Care Dentist 1999;19:164-72.

21. Fellona MO, DeVore LR. Oral health services in primary care nursing centers: opportunities for dental hygiene and nursing collaboration. J Dent Hyg 1999;73:69-77.

22. Chung JP, Mojon P, Budtz-Jørgensen E. Dental care of elderly in nursing homes: perceptions of managers, nurses, and physicians. Spec Care Dentist 2000;20:12-17.

23. Diamond R, Litwak E, Marshall S, et al. Implementing a communitybased oral health care program: lessons learned. $J$ Public Health Dent 2003;63:240-3.

24. dela Cruz GG, Rozier RG, Slade G. Dental screening and referral of young children by pediatric primary care providers. Pediatrics 2004;114:e642-52.

25. Cane RJ, Butler DR. Developing primary health clinical teams for public oral health services in Tasmania. Aust Dent J 2004;49:162-70.

26. Hallberg $U$, Klingberg $G$. Medical health care professionals' assessments of oral health needs in children with disabilities: a qualitative study. Eur J Oral Sci 2005;113:363-8.
27. Maunder PE, Landes DP. An evaluation of the role played by community pharmacies in oral healthcare situated in a primary care trust in the north of England. Br Dent J 2005;199:219-23.

28. Lewis $\mathrm{C}$, Lynch $\mathrm{H}$, Richardson $\mathrm{L}$. Fluoride varnish use in primary care: what do providers think? Pediatrics 2005;115:e69-76.

29. Lowe C, Blinkhorn AS, Worthington HV, et al. Testing the effect of including oral health in general health checks for elderly patients in medical practice - a randomized controlled trial. Community Dent Oral Epidemiol 2007;35:12-17.

30. Andersson K, Furhoff AK, Nordenram G, et al. 'Oral health is not my department'. perceptions of elderly patients' oral health by general medical practitioners in primary health care centres: a qualitative interview study. Scand J Caring Sci 2007;21:126-33.

31. Slade GD, Rozier RG, Zeldin LP, et al. Training pediatric health care providers in prevention of dental decay: results from a randomized controlled trial. BMC Health Serv Res 2007;7:176.

32. Riter D, Maier R, Grossman DC. Delivering preventive oral health services in pediatric primary care: a case study. Health Aff 2008;27:1728-32.

33. Tenenbaum A, Folliguet M, Berdougo B, et al. [Improving the physician-dental surgeon relationship to improve patient care]. Presse Med 2008;37:564-70.

34. Pronych GJ, Brown EJ, Horsch K, et al. Oral health coordinators in long-term care--a pilot study. Spec Care Dentist 2010;30:59-65.

35. Wooten KT, Lee J, Jared $\mathrm{H}$, et al. Nurse practitioner's and certified nurse midwives' knowledge, opinions and practice behaviors regarding periodontal disease and adverse pregnancy outcomes. J Dent Hyg 2011;85:122-31.

36. Skeie MS, Skaret E, Espelid I, et al. Do public health nurses in Norway promote information on oral health? BMC Oral Health 2011;11:1-9.

37. Hajizamani A, Malek Mohammadi T, Hajmohammadi E, et al. Integrating oral health care into primary health care system. ISRN Dent 2012;2012:1-7.

38. Rabiei S, Mohebbi SZ, Patja K, et al. Physicians' knowledge of and adherence to improving oral health. BMC Public Health 2012;12:855-68

39. Brownlee B. Oral health integration in the patient-centered medical home environment: case studies from community health centers. Qualis Health/DentaQuest Foundation 2012 http://docplayer. net/4669109-White-paper-oral-health-integration-in-the-patientcentered-medical-home-pcmh-environment-case-studies-fromcommunity-health-centers.html (accessed 26 Mar 2016).

40. Sams LD, Rozier RG, Wilder RS, et al. Adoption and implementation of policies to support preventive dentistry initiatives for physicians: a national survey of Medicaid programs. Am J Public Health 2013;103:e83-90.

41. Close K, Rozier RG, Zeldin LP, et al. Barriers to the adoption and implementation of preventive dental services in primary medical care. Pediatrics 2010;125:509-17.

42. Olayiwola N, Bodenheimer T, Dubé K, et al. Facilitating care integration in Community Health Centers: a conceptual framework and literature review on best practices for integration into the medical neighborhood. UCSF Center for Excellence in Primary Care 2014 http://www.careinnovations.org/uploads/BSCF_Facilitating_Care Integration_Mar_2014.pdf (accessed 24 Nov 2016).

43. Braimoh M, Ogunbodede E, Adeniyi A. Integration of oral health into primary health care system: views of primary health care workers in Lagos State, Nigeria. J Public Health Afr 2014;5:35-9.

44. Pesaressi E, Villena RS, van der Sanden WJ, et al. Barriers to adopting and implementing an oral health programme for managing early childhood caries through primary health care providers in Lima, Peru. BMC Oral Health 2014;14:17.

45. Mitchell-Royston L, Nowak A, Silverman J. Interprofessional study of oral health in primary care. Pediatric oral health research and policy center 2014 http://www.aapd.org/assets/1/7/Dentaquest_Year_1_ Final_Report.pdf (accessed 15 Nov 2016).

46. Aguiar DM, Tomita NE, Machado MF, et al. Oral health technicians in Brazilian primary health care: potentials and constraints. Cad Saude Publica 2014;30:1560-70.

47. Barnett T, Hoang H, Stuart J, et al. "Sorry, I'm not a dentist": perspectives of rural GPs on oral health in the bush. Med J Aust 2016;204:26.

48. Smith M, Murray-Thomson W. "Not on the radar": dentists perspectives on oral health care of dependent people. Gerodontology 2016:1-11.

49. Arthur T, Rozier RG. Provision of preventive dental services in children enrolled in medicaid by nondental providers. Pediatrics 2016;137:1-10.

50. Langelier M, Moore J, Baker BK, et al. Case studies of 8 federally qualified Health Centers: strategies to integrate Oral Health 
with Primary Care. Rensselaer, NY: Center for Health Workforce Studies, School of Public Health, SUNY Albany, 2015. http://www. oralhealthworkforce.org/wp-content/uploads/2015/11/FQHC-CaseStudies-2015.pdf. (accessed 2 Jan 2017).

51. Bernstein J, Gebel C, Vargas C, et al. Integration of oral health into the well-child visit at federally qualified health centers: study of 6 clinics, August 2014-March 2015. Prev Chronic Dis 2016;13:e58.

52. Tesini DA. Providing dental services for citizens wtih handicaps: a prototype community program. Ment Retard 1987;25:219-22.

53. Nolan L, Kamoie B, Harvey J, et al. The effects of state dental practice laws allowing alternative models of preventive oral health care delivery to low-income children Center for health services research and policy. 2003. https://publichealth.gwu. edu/departments/healthpolicy/DHP_Publications/pub_uploads/ dhpPublication_5F628DDE-5056-9D20-3DFE0BE817BF82D7.pdf (accessed $1 \mathrm{Feb}$ 2017).

54. Rozier RG, Sutton BK, Bawden JW, et al. Prevention of early childhood caries in North Carolina medical practices: implications for research and practice. J Dent Educ 2003;67:876-85.

55. Wysen KH, Hennessy PM, Lieberman MI, et al. Kids get care: integrating preventive dental and medical care using a public health case management model. J Dent Educ 2004;68:522-30.

56. Pan American Health Organisation. 2006. Proposed 10-year regional plan on oral health. USA: WHO. http://iris. paho.org/xmlui/bitstream/ handle/123456789/5685/ce138.r9-e.pdf? sequence=1\&isAllowed $=\mathrm{y}$. (accessed 25 Nov 2016).

57. Heuer S. Integrated medical and dental health in primary care. J Spec Pediatr Nurs 2007;12:61-5.

58. Stevens $\mathrm{J}$, lida $\mathrm{H}$, Ingersoll $\mathrm{G}$. Implementing an oral health program in a group prenatal practice. J Obstet Gynecol Neonatal Nurs 2007;36:581-91.

59. Powell V, Din FM, 2008. Call for an Integrated (Medical/Dental) Health Care Model. Conference on the Electronic Health Record: Best Practices and New Horizons; 1-3 October, 2008. Wake Forest University Translational Science Institute: Winston-Salem, NC, USA. 2008.

60. Weber-Gasparoni K, Kanellis MJ, Qian F. lowa's public health-based infant oral health program: a decade of experience. J Dent Educ 2010;74:363-71.

61. Kruger E, Jacobs A, Tennant M. Sustaining oral health services in remote and indigenous communities: a review of 10 years experience in Western Australia. Int Dent J 2010;60:129-34.

62. Integrated primary and Community Care (IPCC) Development plans for the South West Growth Centre (SWGC). NSW Government 2012 https://www.swslhd.nsw.gov.au/pdfs/SWGC_IPCCS.pdf. (accessed 14 Mar 2016).

63. GrantmakersIn Health. Returning the Mouth to the Body: integrating oral health \& primary care. 2012 http://www.gih.org/ files/FileDownloads/Returning_the_Mouth_to_the_Body_no40_ September_2012.pdf (accessed 13 Dec 2016).

64. U.S. Department of Health and Human Services. Considerations for oral health integration in primary care practice for children. Rockville, Maryland: U.S. Department of Health and Human Services, 2012. https://www.hrsa.gov/oralhealth/oralhealthprimarychildren.pdf. (accessed 14 Feb 2016).

65. NHS Commissioning Board. Securing excellence in commissioning NHS dental services. England: NHS, 2013. https://www.england. nhs.uk/wp-content/uploads/2013/02/commissioning-dental.pdf. (accessed 02 Feb 2017).
66. U.S. Department of Health and Human Services. Integration of Oral Health and Primary Care Practice. Health Resources and Services Administration. 2014 https://www.hrsa.gov/publichealth/clinical/ oralhealth/primarycare/integrationoforalhealth.pdf (accessed $3 \mathrm{Mar}$ 2016).

67. U.S. Department of Health and Human Services. Oral health strategic framework 2014-2017. Public Health Rep 2016;131:242-57.

68. Ramos-Gomez FJ. A model for community-based pediatric oral heath: implementation of an infant oral care program. Int J Dent 2014;2014:1-9.

69. Abrams M, Chung L, Fisher M, et al. San Francisco children's oral health strategic plan 2014-2017. 2014 http://assets.thehcn. net/content/sites/sanfrancisco/Final_document_Nov_2014_ 20141126111021.pdf (accessed 13 Dec 2016).

70. Pucca GA, Gabriel M, de Araujo ME, et al. Ten years of a national oral health policy in Brazil: innovation, boldness, and numerous challenges. J Dent Res 2015;94:1333-7.

71. Pourat N, Martinez AE, Crall JJ. Better together: co-location of dental and primary care provides Opportunities to improve Oral Health. Policy Brief UCLA Cent Health Policy Res 2015;Sep(PB2015-4:1-8.

72. Pucca Junior GA, Lucena EHGde, Cawahisa PT, Pucca G, Gomes de Lucena EH. Financing national policy on oral health in Brazil in the context of the Unified Health System. Braz Oral Res 2010;24(suppl 1):26-32.

73. Leutz WN. Five laws for integrating medical and social services: lessons from the United States and the United Kingdom. Milbank $Q$ 1999;77:77-110.

74. Bloom B, Krathwohl DR. Taxonomy of educational objectives: the classification of educational goals, by a committee of college and university examiners. Handbook I: Cognitive Domain. New York: NY: Longmans, Green, 1956

75. World Health Organization. The World Health report 2008: primary health care now more than ever. 2008 http://www.who.int/whr/2008/ whr08 en.pdf (accessed 12 Apr 2016).

76. Lamarche P, Beaulieu MD, Pineault R, et al. Choices for Change: the path for restructuring primary health care services in Canada: Canadian Foundation for Healthcare Improvement. 2003 http://tools. hhr-rhs.ca/index.php?option=com_mtree\&task=att_download\&link_ id=4431\&cf_id=68\&lang=en (accessed 13 Dec 2016).

77. Titler M. The evidence for evidence-based practice implementation. in: Hughes R, editor. patient safety and quality: an evidence-based handbook for nurses. Rockville: Agency for Healthcare Research and Quality (US), 2008.

78. Kodner D. Introduction to integration. all together now: a conceptual exploration of Integrated Care. Healthcare Quarterly;13:6-15.

79. Tsasis P, Evans JM, Owen S. Reframing the challenges to integrated care: a complex-adaptive systems perspective. Int J Integr Care 2012;12.

80. Pomerantz AS, Corson JA, Detzer MJ. The challenge of integrated care for mental health: leaving the 50 minute hour and other sacred things. J Clin Psychol Med Settings 2009;16:40-6.

81. Dickinson $\mathrm{H}$, Glasby RMandJ. The challenges of delivering integration. J Integr Care 2014;22.

82. Lapkin S, Levett-Jones T, Gilligan C. A systematic review of the effectiveness of interprofessional education in health professional programs. Nurse Educ Today 2013;33:90-102.

83. Shrader S, Kostoff M, Shin T, et al. Using communication technology to enhance interprofessional education simulations. Am J Pharm Educ 2016;80:13. 